\title{
Covariant perturbation theory and the Randall-Sundrum picture
}

\author{
Ezequiel Alvarez ${ }^{\mathrm{a}}$, Francisco D. Mazzitelli ${ }^{\mathrm{b}}$ \\ a Instituto Balseiro, Centro Atómico Bariloche, 8400 Bariloche, Argentina \\ ${ }^{\mathrm{b}}$ Departamento de Física, Facultad de Ciencias Exactas y Naturales, Universidad de Buenos Aires, Ciudad Universitaria, \\ Pabellón I, 1428 Buenos Aires, Argentina
}

Received 27 October 2000; received in revised form 20 December 2000; accepted 21 February 2001

Editor: H. Georgi

\begin{abstract}
The effective action for quantum fields on a $d$-dimensional spacetime can be computed using a non-local expansion in powers of the curvature. We show explicitly that, for conformal fields and up to quadratic order in the curvature, the non-local effective action is equivalent to the $d+1$ action for classical gravity in $\operatorname{AdS}_{d+1}$ restricted to a $(d-1)$-brane. This generalizes previous results about quantum corrections to the Newtonian potential and provides an alternative method for making local a non-local effective action. The equivalence can be easily understood by comparing the Kallen-Lehmann decomposition of the classical propagator with the spectral representation of the non-local form factors in the quantum effective action. ๑ 2001 Published by Elsevier Science B.V.
\end{abstract}

1. The analysis of the physical effects of quantum fields on the background geometry requires the calculation of the effective action. This is a complicated object even for free fields. With the exception of a few highly symmetric background metrics, it cannot be computed exactly. Moreover, in order to study problems like black hole evaporation or the physics of the early universe, it is necessary to compute the effective action for an arbitrary metric, that should be fixed at the end by minimizing the effective action.

A useful approach for the approximate computation of the effective action is the so-called covariant perturbation theory [1]. In this approach, that can be understood as a summation the Schwinger-DeWitt expansion, the effective action is written in powers of curvatures. This approximation contains non-local terms that include important physical information like gravitational particle creation and the leading long distance quantum corrections to general relativity.

For conformal fields, in two spacetime dimensions the quadratic term in the covariant perturbation theory reproduces the (exact) Polyakov action. It is possible to derive Hawking radiation from it [2]. In four dimensions this has still not been done, and indeed it is a very difficult task because Hawking radiation is contained in the cubic terms of the expansion.

E-mail addresses: sequi@df.uba.ar (E. Alvarez), fmazzi@df.uba.ar (F.D. Mazzitelli). 
The four-dimensional quadratic effective action has been used to compute the leading long distance $1 / r^{3}$ corrections to the Newtonian potential [3]

$$
V(r)=-\frac{G M}{r}\left(1-\frac{(16 \pi)^{2} G B_{4}}{r^{3}}\right),
$$

where the constant $B_{4}$ depends on the spin and number of quantum matter fields. These corrections have been computed by other methods a long time ago [4]. For related works see [5].

In a recent paper, Duff and Liu [6] proved that the same kind of corrections to the gravitational potential do appear in the Randall-Sundrum brane-world proposal [7]. When a 3-brane is inserted into $\mathrm{AdS}_{5}$, and for classical matter fields in the brane, the classical metric in five dimensions restricted to the brane reproduces the classical Newtonian potential plus the $1 / r^{3}$ corrections. The coefficient of $1 / r^{3}$ that appears in this scenario coincides with the coefficient due to closed loops of $\mathcal{N}=4$ superconformal $U(N)$ Yang-Mills theory in the four-dimensional theory. This is in tune with the AdS/CFT correspondence [8].

In this Letter we will extend the results of Ref. [6]. We will prove that, up to quadratic order in the curvature and for free conformal fields, the non-local $d$-dimensional effective action coincides with the restriction of the gravitational action in $\operatorname{AdS}_{d+1}$ to a $(d-1)$-brane. The results are valid for $d>2$. We stress that we are not trying to check the consistency between the AdS/CFT and the brane-world relations, as in Ref. [6]. Our aim is to provide an alternative representation for the non-local $d$-dimensional effective action.

2. For a scalar field in curved spacetimes the effective action is given by $\Gamma=\frac{1}{2} \ln \operatorname{det}\left(\mathcal{O} / \mu^{2}\right)$, where $\mathcal{O}=-g^{\mu \nu} \nabla_{\mu} \nabla_{\nu}+m^{2}+\xi R$ is the operator of the classical field equation, $\mu$ is an arbitrary parameter with dimensions of mass, $m$ is the mass of the scalar field, and $\xi$ is the coupling to the scalar curvature. The conformal coupling in $d$ dimensions is $\xi=\xi_{c}=\frac{1}{4} \frac{d-2}{d-1}$.

Using heat kernel techniques [9] it is possible to obtain the Schwinger-DeWitt expansion for the effective action

$$
\Gamma=-\frac{1}{2(4 \pi)^{d / 2}} \int_{0}^{\infty} \frac{d s}{s^{1+\frac{d}{2}}} e^{-m^{2} s} \sum_{l \geqslant 0} \frac{(-s)^{l}}{l !} \int d^{d} x \sqrt{g} a_{l}(x) .
$$

The Schwinger-DeWitt coefficients $a_{l}$ are functions of the curvature and its covariant derivatives. When integrating out term by term the expression above, an expansion in inverse powers of the mass is obtained. The expansion is valid for slowly varying metrics that satisfy $\mathcal{R} \ll m^{2}$ ( $\mathcal{R}$ denotes components of the curvature tensor). The expansion is local, and adequate for the analysis of the divergences of the theory, which are contained in the terms with $l$ less or equal to the integer part of $d / 2$. However, it misses very important physical effects (like particle creation), and it is not adequate for massless quantum fields.

It is possible to perform a partial summation of the Schwinger-DeWitt expansion by keeping terms up to a given order in the curvature. The idea was introduced in Ref. [10] and further developed in Refs. [1,11]. The effective action for a massless scalar field in $d$ spacetime dimensions, up to quadratic order in the curvature, can be written as [11]

$$
\Gamma=\Gamma_{\text {local }}+\Gamma_{\text {nonloc }},
$$

where

$$
\Gamma_{\text {local }}=\int d^{d} x \sqrt{-g}\left[-\Lambda_{(d)}+M_{(d)}^{d-2} R_{(d)}-\sum_{l=0}^{k}\left(\alpha^{(l)} R_{(d)} \square^{l} R_{(d)}+\beta^{(l)} R_{\mu \nu(d)} \square^{l} R_{(d)}^{\mu \nu}\right)\right]
$$

and

$$
\Gamma_{\text {nonloc }}=-\alpha \int d^{d} x \sqrt{-g}\left(a R_{(d)} f(\square) R_{(d)}+b R_{\mu \nu(d)} f(\square) R_{(d)}^{\mu \nu}\right) .
$$


Here $g_{\mu \nu}, x^{\mu}, M_{(d)}$ and $\Lambda_{(d)}$ are the $d$-dimensional metric, coordinates, Planck mass $\left(M_{(4)}^{2}=1 / 16 \pi G_{\text {Newton }}\right)$ and cosmological constant, $R_{(d)}$ and $R_{\mu \nu(d)}$ the Ricci tensor and scalar, respectively,

$$
\begin{aligned}
& \alpha=(4 \pi)^{-d / 2} \frac{\sqrt{\pi}}{8 \Gamma((d-1) / 2)}, \\
& a=\left(\xi-\xi_{c}\right)^{2}-\frac{d}{8(d-1)^{2}(d+1)}, \quad b=\frac{1}{2\left(d^{2}-1\right)},
\end{aligned}
$$

and

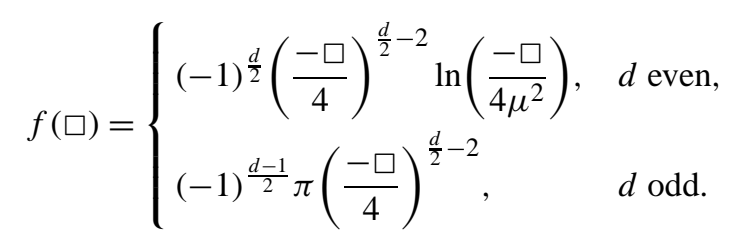

The summation in Eq. (4) runs up to $k$, the integer part of $d / 2-2$. These terms are needed to renormalize the theory. After renormalization, the coefficients $\alpha^{(l)}, \beta^{(l)}$ and $\Lambda_{(d)}$ might take arbitrary values. For simplicity we will take $\Lambda_{(d)}=0$ in what follows.

The results for the effective action can be extended for fields of arbitrary spin [11]. For example, for a massless Dirac field in four dimensions, the effective action is six times the result for a conformally coupled scalar field [12].

For $g_{\mu \nu}=\eta_{\mu \nu}+h_{\mu \nu}$, and in the harmonic gauge (i.e., $h_{\mu \nu}{ }^{\nu}=\frac{1}{2} h_{\alpha}{ }^{\alpha}, \mu$ ), Eq. (3) can be rewritten as

$$
\Gamma^{(2)}=\frac{-1}{4} \int d^{d} x h_{\mu \nu}\left(\Delta^{-1}\right)_{\rho \sigma}^{\mu \nu} h^{\rho \sigma}
$$

where

$$
\begin{aligned}
\left(\Delta^{-1}\right)_{\rho \sigma}^{\mu \nu}= & -M_{(d)}^{d-2} \square\left(\eta_{\rho}^{\mu} \eta_{\sigma}^{\nu}-\frac{1}{2} \eta^{\mu v} \eta_{\rho \sigma}\right)+\alpha \square^{2} f(\square)\left(b \eta_{\rho}^{\mu} \eta_{\sigma}^{v}+a \eta^{\mu v} \eta_{\rho \sigma}\right) \\
& +\sum_{l=0}^{k} \square^{l+2}\left(\alpha^{(l)} \eta^{\mu v} \eta_{\rho \sigma}+\beta^{(l)} \eta_{\rho}^{\mu} \eta_{\sigma}^{v}\right) .
\end{aligned}
$$

If we add to the theory classical matter described by an energy-momentum tensor $T^{\mu \nu}$, the spacetime metric satisfies

$$
h_{\mu \nu}=\Delta_{\mu \nu}^{\alpha \beta} T_{\alpha \beta} .
$$

In the low-energy approximation the quantum correction in Eq. (7) can be treated as a small perturbation. Using that the inverse of $A \eta^{\mu \nu} \eta_{\rho \sigma}+B \eta_{\rho}^{\mu} \eta_{\sigma}^{\nu}$ is given by $\frac{-A}{B(B+A \cdot d)} \eta^{\alpha \beta} \eta_{\mu \nu}+\frac{1}{B} \eta_{\mu}^{\alpha} \eta_{\nu}^{\beta}$, it is straightforward to check that, up to leading order,

$$
\begin{aligned}
\Delta_{\mu \nu}^{\alpha \beta}= & \frac{-1}{M_{(d)}^{d-2} \square}\left(\eta_{\mu}^{\alpha} \eta_{\nu}^{\beta}-\frac{1}{d-2} \eta^{\alpha \beta} \eta_{\mu \nu}\right) \\
& -\frac{\alpha}{2\left(d^{2}-1\right) M_{(d)}^{2(d-2)}} f(\square)\left(\eta_{\mu}^{\alpha} \eta_{\nu}^{\beta}-\left[\frac{1}{d-1}-\left(\xi-\xi_{c}\right)^{2} 8 \frac{d^{2}-1}{(d-2)^{2}}\right] \eta^{\alpha \beta} \eta_{\mu \nu}\right) \\
& +\sum_{j=0}^{\tilde{k}} g_{j}^{(1)} \square^{j} \eta_{\mu}^{\alpha} \eta_{\nu}^{\beta}+g_{j}^{(2)} \square^{j} \eta^{\alpha \beta} \eta_{\mu \nu} .
\end{aligned}
$$


The constants $g_{j}^{(i)}$ depend on $d, M_{(d)}, \alpha^{(l)}$ and $\beta^{(l)}$. The summation here and in what follows is only for $d \geqslant 5$; $\tilde{k}$ is equal to $k$ when $d$ is odd and to $k-1$ when $d$ is even.

The meaning of Eq. (9) is very simple: the first term corresponds to the classical propagation while the second contains the quantum corrections and is easily traced back to the non-local part of the action, Eq. (5). The analytic terms (proportional to $\square^{j}$ ), will not contribute in the large distance/low-energy limit (see below).

3. We will now prove that a propagator similar to Eq. (9) describes the classical propagation on a brane inserted into $\mathrm{AdS}_{d+1}$ [13]. If the $d$-dimensional spacetime is thought as a $(d-1)$-brane in a $(d+1)$-dimensional theory then the classical action reads

$$
\int d^{d+1} X \sqrt{-G}\left(M_{(d+1)}^{d-1} R_{(d+1)}-\Lambda_{(d+1)}+\mathcal{L}_{\text {matter }}\right)-\int d^{d} x \sqrt{-g} \tau .
$$

Here $X^{I}=\left(y, x^{\rho}\right)$ are the $(d+1)$-dimensional coordinates, $G_{I J}$ the metric in $d+1$ dimensions, and $\tau$ the brane tension. The $\mathcal{L}_{\text {matter }}$ term may include a matter source in the brane as well as in the bulk. The brane geometry is chosen such that $y$ is the coordinate in the bulk and $x^{\rho}$ are coordinates along the brane (which is located at $y=0$ ), then small fluctuations to the metric are represented by

$$
d s^{2}=d y^{2}+e^{-2|y| / L}\left(\eta_{\mu \nu}+h_{\mu \nu}\left(x^{\rho}, y\right)\right) d x^{\mu} d x^{\nu},
$$

where $L=\sqrt{-d(d-1) M_{(d+1)}^{d-1} / \Lambda_{(d+1)}}$.

We are only interested in $h_{\mu \nu}\left(x^{\rho}, y=0\right)$ when the matter source is located on the brane. In this situation, it has been shown that the effective propagator on the brane is given by [13]

$$
\Delta_{\mu \nu}^{\alpha \beta}=-\frac{d-2}{L M_{(d+1)}^{d-1}} \frac{1}{\square}\left(\eta_{\mu}^{\alpha} \eta_{\nu}^{\beta}-\frac{1}{d-2} \eta^{\alpha \beta} \eta_{\mu \nu}\right)-\frac{1}{M_{(d+1)}^{d-1}} \Delta_{\mathrm{KK}}(\sqrt{-\square})\left(\eta_{\mu}^{\alpha} \eta_{\nu}^{\beta}-\frac{1}{d-1} \eta^{\alpha \beta} \eta_{\mu \nu}\right),
$$

where

$$
\Delta_{\mathrm{KK}}(\sqrt{-\square})=\frac{-1}{\sqrt{-\square}} \frac{K_{d / 2-2}(\sqrt{-\square} L)}{K_{d / 2-1}(\sqrt{-\square} L)} .
$$

Again Eq. (12) has a simple interpretation: the first term describes the zero mode graviton localized on the brane, while the second term corresponds to the continuum Kaluza-Klein graviton modes.

At large distances, corresponding to $\sqrt{-\square} L \ll 1$, Eq. (13) can be expanded to give, up to the first term nonanalytic-in- $\square$,

$$
\frac{1}{L} \Delta_{\mathrm{KK}}(\sqrt{-\square}) \approx\left\{\begin{array}{l}
\sum_{l=0}^{\tilde{k}} c_{l}^{(e)}\left(\square L^{2}\right)^{l}+c_{d / 2-2}^{(e)}(-1)^{\frac{d}{2}}\left(\frac{-\square}{4} L^{2}\right)^{\frac{d}{2}-2} \ln \left(-\square L^{2}\right), \quad d \text { even }, \\
\tilde{k} c_{l=0}^{(o)}\left(\square L^{2}\right)^{l}+\pi c_{d / 2-2}^{(o)}(-1)^{\frac{d-1}{2}}\left(\frac{-\square}{4} L^{2}\right)^{\frac{d}{2}-2}, \quad d \text { odd } .
\end{array}\right.
$$

The coefficients $c_{l}^{(i)}$ can be easily obtained, but we will not need the explicit expression in what follows.

Now we compare Eqs. (12) and (9). The classical terms in both propagators coincide if we choose the coupling constants such that $\frac{d-2}{L M_{(d+1)}^{d-1}}=\frac{1}{M_{(d)}^{d-2}}$. In order to have agreement between the leading non-analytic terms, the coupling must be conformal, i.e., $\xi=\xi_{c}$. Moreover, we must have $c_{d / 2-2}^{(e, o)} L^{d-3} / M_{(d+1)}^{d-1}=\alpha\left(2\left(d^{2}-1\right) M_{(d)}^{2(d-2)}\right)^{-1}$. These equations relate the values of the $d+1$ cosmological constant and Planck mass with Planck mass in $d$ dimensions. Had we considered a different free field content on the brane ( $N_{s}$ fields of spin $\left.s\right)$ the only difference would have been a different relation between the values of these parameters. 
It is not necessary to require agreement between the terms analytic in $\square$ since, as shown below, they are not relevant in the low-energy limit. However, it is worth noting that with the choice $g_{j}^{(2)}=-\frac{1}{d-1} g_{j}^{(1)}$ and $g_{j}^{(1)}=-c_{j}^{(e, o)} L^{2 j+1} / M_{(d+1)}^{d-1}$ the analytic terms also coincide. This would imply particular values for the constants $\alpha^{(l)}$ and $\beta^{(l)}$ in the non-local effective action Eq. (5), all of them determined by $M_{(d+1)}$ and $\Lambda_{(d+1)}$.

We will now show that analytic terms are not relevant in the low-energy limit. To illustrate this point we compute the quantum corrections to the $d$-dimensional Newtonian potential, $-\frac{1}{2} h^{00}(x)$. We assume a classical mass $M$ fixed at the origin of coordinates, namely $T^{\mu \nu}(x)=\delta_{0}^{\mu} \delta_{0}^{\nu} M \delta^{(d-1)}(\vec{x})$. Here $\vec{x}$ are the space coordinates, $\vec{x}=\left(x_{1}, x_{2}, \ldots, x_{d-1}\right)$. With this in mind, using Eqs. (8) and (9), the quantum corrected Newtonian potential reads ${ }^{1}$

$$
V(r)=\frac{-1}{2} h^{00}(r)=\left\{\begin{array}{l}
B_{3} \frac{M}{M_{(3)}^{2}} \frac{1}{r}, \quad d=3, \\
A_{d} \frac{M}{M_{(d)}^{d-2}} \frac{1}{r^{d-3}}+B_{d} \frac{M}{M_{(d)}^{2(d-2)}} \frac{1}{r^{2 d-5}}-\frac{M}{2} \sum_{j=0}^{\tilde{k}}\left(g_{j}^{(1)} \square^{j}+g_{j}^{(2)} \square^{j}\right) \delta^{(d-1)}(\vec{x}), \quad d \geqslant 4,
\end{array}\right.
$$

where $A_{d}$ and $B_{d}$ are constants and $r=|\vec{x}|$. As anticipated, the analytic terms proportional to $\square^{j}$ produce quantum corrections localized at the origin. They are therefore irrelevant at large distances. In four dimensions, the Newtonian potential reads

$$
V(r)=-\frac{G M}{r}\left[1+\frac{G}{45 \pi r^{2}}\left(1+45\left(\xi-\frac{1}{6}\right)^{2}\right)\right],
$$

and agrees with previous results for $\xi=1 / 6$ [6,12]. If we consider $N_{0}$ scalar fields and $N_{1 / 2}$ Dirac fields, the Newtonian potential becomes

$$
V(r)=-\frac{G M}{r}\left\{1+\frac{G}{45 \pi r^{2}}\left[N_{0}\left(1+45\left(\xi-\frac{1}{6}\right)^{2}\right)+6 N_{1 / 2}\right]\right\} .
$$

4. Non-local effective actions have been previously localized through the introduction of auxiliary fields. For example, in two dimensions, Polyakov's action

$$
S_{P}=-\frac{1}{96 \pi} \int d^{2} x \sqrt{-g} R \frac{1}{\square} R
$$

can be made local by introducing an auxiliary field $\psi$ and the local action

$$
S_{\text {local }}=-\frac{1}{96 \pi} \int d^{2} x \sqrt{-g}(-\psi \square \psi+2 \psi R) .
$$

On shell for the auxiliary field, both actions $S_{P}$ and $S_{\text {local }}$ are equivalent.

In four dimensions, the effective action that reproduces the conformal anomaly is the so-called Reigert's action [14]. The non-local part of the Reigert's action is, schematically,

$$
S_{R}=\int d^{4} x \mathcal{R}^{2} \frac{1}{\Delta_{4}} \mathcal{R}^{2},
$$

where $\mathcal{R}$ denotes components of the Riemann tensor, and $\Delta_{4}$ is the fourth-order operator

$$
\Delta_{4}=\square^{2}-2 R^{\mu \nu} \nabla_{\mu} \nabla_{\nu}+\frac{2}{3} R \square-\frac{1}{3} \nabla^{\mu} R \nabla_{\mu}
$$

\footnotetext{
1 Note that for $d=3$ spacetime is flat outside matter, hence there is no gravitational force. The term proportional to $B_{3}$ comes from the quantum correction.
} 
Reigert's action can be made local $[14,15]$ by the introduction of auxiliary scalar fields

$$
S_{\text {local }}=\int d^{4} x\left(-\psi \Delta_{4} \psi+2 \psi \mathcal{R}^{2}\right) .
$$

The localization based in the introduction of auxiliary fields works only when the form factors in the non-local effective action are the inverse of polynomials in $\square$ and $\nabla_{\mu}$. Here the form factors does not satisfy this property. An extra dimension is needed to make local the action. The mathematical reason for this can be understood as follows. The non-analytic form factors can be represented in the form of spectral integrals [1]. For example, in three and four dimensions the form factors can be written as

$$
\ln \left(\frac{-\square}{\mu^{2}}\right)=\int_{0}^{\infty} d \lambda\left(\frac{1}{\lambda-\square}-\frac{1}{\lambda+\mu^{2}}\right), \quad(-\square)^{-1 / 2}=\frac{2}{\pi} \int_{0}^{\infty} d \lambda\left(\frac{1}{\lambda^{2}-\square}\right) .
$$

Similar expressions can be found for other dimensions. Note that the non-analytic functions of $\square$ are written as integrals that involve massive propagators $\left(\frac{1}{\lambda-\square}\right.$ or $\left.\frac{1}{\lambda^{2}-\square}\right)$.

On the other hand, the restriction of a massless $d+1$ propagator on a $(d-1)$-brane also admits an analogous representation [16]. Indeed, let us consider the metric $d s^{2}=d y^{2}+w^{2}(y) g_{\mu \nu}(x) d x^{\mu} d x^{\nu}$. The D'Alambertian operator can be written as

$$
\square_{d+1}=\frac{\square}{w^{2}}+\frac{\partial^{2}}{\partial y^{2}}+d \frac{w^{\prime}}{w} \frac{\partial}{\partial y} \equiv \frac{\square}{w^{2}}+\square_{y},
$$

where $\square$ is the $d$-dimensional D'Alambertian associated to $g_{\mu \nu}$.

We introduce the eigenfunctions $\theta_{\lambda}^{(i)}(y)$, that satisfy $\square_{y} \theta_{\lambda}^{(i)}=-\frac{\lambda}{w^{2}} \theta_{\lambda}^{(i)}$. It can be easily shown [16] that the massless propagator $\Delta=\frac{1}{\square_{d+1}}$, restricted to a fixed slice $y=$ const, admits the following representation

$$
\Delta\left(x, y, x^{\prime}, y\right)=\sum_{i, \lambda}\left|\theta_{\lambda}^{(i)}(y)\right|^{2} \frac{1}{\square-\lambda} .
$$

This is analogous to the Kallen-Lehman decomposition in quantum field theory, with a weight function $\mu(\lambda, y)=$ $\sum_{i}\left|\theta_{\lambda}^{(i)}(y)\right|^{2}$.

The similarity between the form factors in the non-local quantum effective action and the restriction of the classical propagator on a brane is now clear (compare Eqs. (21) and (23)). Roughly speaking, in this Letter we have shown that the weight function in $\mathrm{AdS}_{d+1}$ spacetime reproduces the spectral representation of the $d$-dimensional form factor for conformal fields. It is possible that, by taking a different metric in the bulk, one could reproduce the non-local effective action for non-conformal fields. Alternatively, a different quantum field theory on the brane could reproduce the $\mathrm{AdS}_{d+1}$ propagator beyond leading order.

The equivalence shown in this Letter could be useful as a tool for computations of the effects of quantum fields on the spacetime metric, since it may be technically more easy to work with an extra dimension than with a non-local effective action.

\section{Acknowledgements}

This work was supported by Universidad de Buenos Aires, CONICET (Argentina) and CNEA (Argentina). We would like to thank G. Giribet and J. Russo for useful conversations. 


\section{References}

[1] A.O. Barvinsky, G.A. Vilkovisky, Nucl. Phys. 333 (1990) 471.

[2] V.P. Frolov, G.A. Vilkovisky, in: M.A. Markov, P.C. West (Eds.), Quantum Gravity, Plenum, London, 1984.

[3] D. Dalvit, F.D. Mazzitelli, Phys. Rev. D 50 (1994) 1001.

[4] M.J. Duff, Phys. Rev. D 9 (1974) 1837.

[5] J.F. Donoghue, Phys. Rev. Lett. 72 (1994) 2996;

H. Hamber, S. Liu, Phys. Lett. B 357 (1995) 51;

I. Muzinich, S. Vokos, Phys. Rev. D 52 (1995) 3472.

[6] M.J. Duff, J.T. Liu, Phys. Rev. Lett. 85 (2000) 2052.

[7] L. Randall, R. Sundrum, Phys. Rev. Lett. 83 (1999) 4690.

[8] J.M. Maldacena, Adv. Theor. Math. Phys. 2 (1998) 231;

O. Aharony et al., Phys. Rep. 323 (2000) 183.

[9] J.S. Schwinger, Phys. Rev. 82 (1951) 664;

B.S. DeWitt, Dynamical Theory of Groups and Fields, Gordon and Breach, New York, 1965.

[10] G.A. Vilkovisky, in: S.M. Christensen (Ed.), Quantum Theory of Gravity, Hilger, Bristol, 1984.

[11] I.G. Avramidy, Sov. J. Nucl. Phys. 49 (1989) 735.

[12] D. Dalvit, PhD Thesis, University of Buenos Aires, hep-th/9807112.

[13] S.B. Giddings, E. Katz, L. Randall, JHEP 0003 (2000) 023.

[14] R.J. Reigert, Phys. Lett. B 134 (1984) 56.

[15] I.L. Shapiro, A.G. Jacksenaev, Phys. Lett. B 324 (1994) 284;

R. Balbinot, A. Fabbri, I. Shapiro, Nucl. Phys. B 559 (1999) 301.

[16] M. Bertola, J. Bros, V. Gorini, U. Moschella, R. Schaeffer, hep-th/0003098. 